\title{
Control Theory and Technology Robot Impedance Control and Passivity Analysis with Inner Torque and Velocity Feedback Loops
}

\author{
Michele Focchi ${ }^{1 \dagger}$, Gustavo A. Medrano-Cerda ${ }^{1}$, Thiago Boaventura ${ }^{2}$, Marco Frigerio ${ }^{1}$, Claudio Semini ${ }^{1}$, \\ Jonas Buchli ${ }^{2}$, Darwin G. Caldwell ${ }^{1}$, \\ 1. Dept. of Advanced Robotics, Istituto Italiano di Tecnologia (IIT), via Morego, 30, 16163 Genova, Italy; \\ 2. Agile \& Dexterous Robotics Lab, ETH Zürich, Tannenstr. 3, 8092 Zürich, Switzerland; \\ Received 21st January 2015; revised 19th October 2015; accepted 22nd October 2015
}

\begin{abstract}
:
Impedance control is a well-established technique to control interaction forces in robotics. However, real implementations of impedance control with an inner loop may suffer from several limitations. In particular, the viable range of stable stiffness and damping values can be strongly affected by the bandwidth of the inner control loops (e.g. a torque loop) as well as by the filtering and sampling frequency. This paper provides an extensive analysis on how these aspects influence the stability region of impedance parameters as well as the passivity of the system. This will be supported by both simulations and experimental data. Moreover, a methodology for designing joint impedance controllers based on an inner torque loop and a positive velocity feedback loop will be presented. The goal of the velocity feedback is to increase (given the constraints to preserve stability) the bandwidth of the torque loop without the need of a complex controller.
\end{abstract}

Keywords: Impedance control, torque control, passivity and stability analysis

DOI $10.1007 / s 11768-014-* * * * * *$

\section{Introduction}

Until recently, the majority of legged robots employed high-gain (stiff) position feedback control [1]. However, this approach is unsuitable when a robot is in contact with unstructured real-world environment, as the controller would try to satisfy the position goal at all costs [2]. Instead, for such scenarios, a force/torque control in joint or end-effector space is desirable.

For a legged robot, force control can be useful in both the swing and stance phase. During stance, it allows to control the ground impact forces, with the purpose to improve balance capabilities. During the swing phase, it plays a crucial role in providing to the robot's leg the compliance necessary to negotiate unperceived obstacles, while still ensuring a good position tracking by using rigid body inverse dynamics. Interaction forces can be regulated in two ways: passively and actively. Passive methods are those in which physical compliant elements are included be- tween the robot and the environment to limit the interaction forces (e.g. a passive spring in series elastic actuators [3], [4]). On the other hand active compliance is achieved through the active control of joints (position or torque) using feedback measurements of joint torques [5]. This can emulate a virtual compliance both at the joint as well as at the end-effector/foot level.

A major benefit of active compliance is its ability to change the dynamic characteristics (e.g. stiffness and damping) in real-time. Hence, legged robots can take advantage of active compliance to adapt the leg stiffness to swing and stance phases, or to the surface properties [6]. Many methods to actively control compliance at the endeffector have been developed, such as impedance control [7], operational space control [8], hybrid force-control [9], and virtual model control [10]. Impedance control, in particular, allows the dynamic characteristics at the robot interaction port (e.g. the end-effector) to be specified by reg-

${ }^{\dagger}$ Corresponding author.

E-mail: michele.focchi@iit.it Tel.: +39 010 71781974; fax: +39 01071781232.

This work was supported by the Istituto Italiano di Tecnologia.

(C) 2015 Istituto Italiano di Tecnologia, Advanced Robotics Department, Italy. 
ulating the dynamic relationship between forces and positions (mechanical impedance). Despite impedance is of primary importance to achieve dynamically stable robot locomotion, only recently an exhaustive research has been carried out, on the MIT Cheetah robot, to find which impedance parameters are suitable for locomotion [11]. However, an analysis that investigates if these parameters are realizable is still missing.

In the past, impedance control algorithms were limited by the controller bandwidth, which was set by the computational power and actuator dynamics. That was one of the reasons for the introduction of passive elements in series with the actuator [12], which have intrinsically unlimited bandwidth. However, recent advances in both computer and actuator performance, made active compliance feasible for highly-dynamic applications [13,14]. Nevertheless, many aspects, still create stability issues on impedance control. For instance, the range of stable stiffness and dampings that can be virtually created (Z-width [15], where $Z$ stands for impedance [16]) can be limited by filtering, sampling frequency, and also by the bandwidth of inner control loops (e.g. a torque loop).

A common practice in designing nested loop control systems is to maximize the bandwidth of the innermost loop [17]. However, maximizing the inner loop controller bandwidth is not always the best strategy. When the outer impedance loop is closed, designing the inner loop to have the highest possible bandwidth reduces the range of impedance parameters for which the whole system is stable, as demonstrated later in this work. Therefore, a tradeoff must be found between: having a high bandwidth to ensure good torque and impedance tracking, and keeping the bandwidth low to increase the range of stable impedance values. Other aspects that directly influence the stability region are the sampling frequency and filtering [18]. Their effect is to introduce delays into the control loop, and their influence will also be investigated in this work. To ensure closed-loop stability during interactions with the environment or other systems, the controller must be designed to ensure the system behaves passively at the interaction port [19], [20]. From the passivity property, asymptotic stability can always be ensured: both in free motion as well as when the robot is in contact with any type of environment (which is usually passive). Physical compliant elements and rigid bodies are passive by nature. However, when the compliant behavior is emulated by an actuator, the passivity is a function of the controller gains. In this work it will be shown that passivity can also be a restrictive condition to select impedance parameters.
Related works. The published literature about active compliance is vast. A brief review on the issues that affect the performance of force controlled robots can be found in [21]. Stability analysis and performance specifications for compliance control was first introduced by Kazerooni et al. [22] for a manipulator whose model had bounded uncertainty. Lawrence in [23] considers the non-ideal, practical effects of computation and communication delays on impedance control and finds some stability boundaries. However, his analysis was in continuous time and it is not necessarily valid for discrete time systems. Indeed sampling is not completely equivalent to time delays because when sampling there are additional zeros that do not appear in continuous time.

Regarding controllers based on passivity, Albu-Schaffer et al. in [20] implemented a full state controller for joint or Cartesian impedance with passive capabilities. The controller is not passive itself but it is together with the motor dynamics. The torque feedback shapes the rotor inertia of the motors to a desired value. More recently Buerger and Hogan [24] have revisited the problem of designing controllers for physically interactive robots. For a $1 \mathrm{DoF}$ system, they reformulated the problem as a robust stability problem based on $\mu$-synthesis (structured singular values) and loop shaping methods. The approach provides improvements in robot performance compared to traditional passive controllers. In [25] stiffness and impedance control concepts were used for robot-aided rehabilitation. New stability conditions were proposed using Lyapunov approach and based on the relationship between the dynamics of the robot and its energy. In [26] Yasrebi et al. carried out a time-domain passivity analysis of the Z-width diagram. This led to the design of a new haptic controller which extended the range of stable impedance parameters (Zwidth) by means of an acceleration feedback. The analysis was carried out for one joint using passivity theory in the frequency domain.

The main contribution of this work, is a methodology to analyze (based on an accurate model) stability and passivity of a gearbox driven actuator (plus load) system. The analysis takes into account all the non-idealities present in real implementation of an impedance controller, namely: actuator dynamics, discrete implementation, filtering, nested loops. This allows to find the impedance "stability regions" which represent the impedance parameters that can be rendered in a stable way. Simulations and experimental data show how the above-mentioned nonidealities influence the stability regions as well as the passivity of the system. The study is carried out for the ad- 
duction/adduction $(H A A)$ joint of the HyQ [27] robot (see Fig. 1), where impedance control was implemented with an inner torque loop [28]. However, the underlying ideas are valid for any electric actuator moving a load with a gearbox reduction. In the bigger picture, the stability regions are the basis to develop a gain scheduler (in the low-level control layer) which is able to adapt the bandwidth of the inner torque loop according to the impedance parameters set by the user.

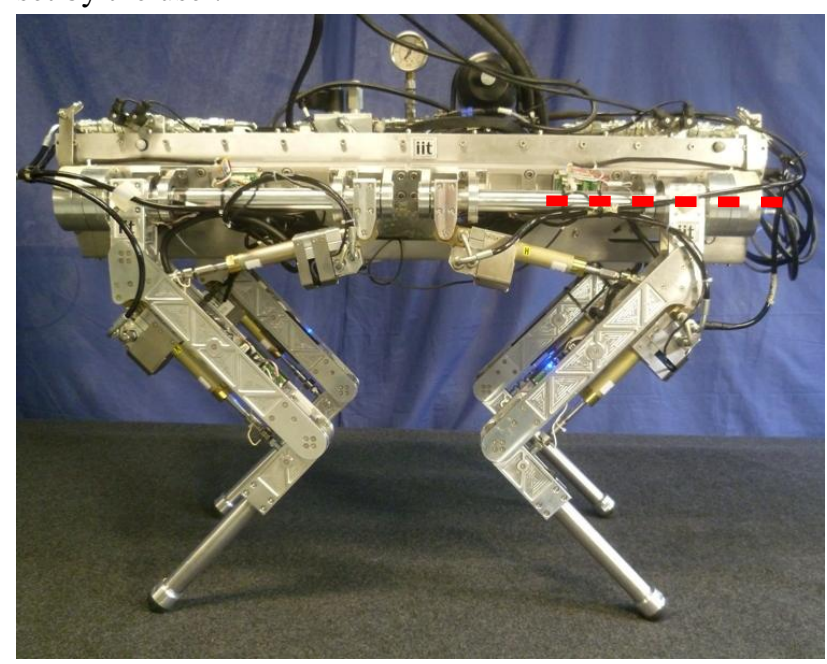

Fig. 1: Picture of a lateral view of the HyQ robot, the HAA joint axis is marked in red.

This paper is structured as follows: the mathematical model of the system is introduced in Section 2 followed by a description of the control system implementation in Section 3 . The stability issues associated with real implementation of an impedance controller are analysed, both in simulations and experimentally, in Section 4. A brief assessment about passivity for the system is then given in Section 5. Finally, Section 6 discusses the results and future works.

\section{System description and mathematical model}

The studies and experiments presented in this work are all conducted on HyQ [27]. HyQ is a fully torquecontrolled quadruped robot with a mix of hydraulic and electric actuation for each leg: two hydraulic joints on the sagittal plane (hip HFE and knee KFE flexion-extension) and one electric joint moving in the traversal plane (hip adduction-abduction HAA, Fig.2 on the left). This paper focuses on modeling and control of the electric joint, which consists of a DC brush-less motor (Emoteq HT2301) and a harmonic drive gearbox (CSD-25-100). The leg is attached to the gearbox output via an interface consisting of 6 parallel pins (evenly distributed on a circle around the axis of rotation) that enable easy dismounting (see Fig. 3). This interface represents a small intermediate rotational inertia $\left(J_{L 1}\right)$ placed before the inertia represented by the leg $\left(J_{L 2}\right)$ in the transmission train.

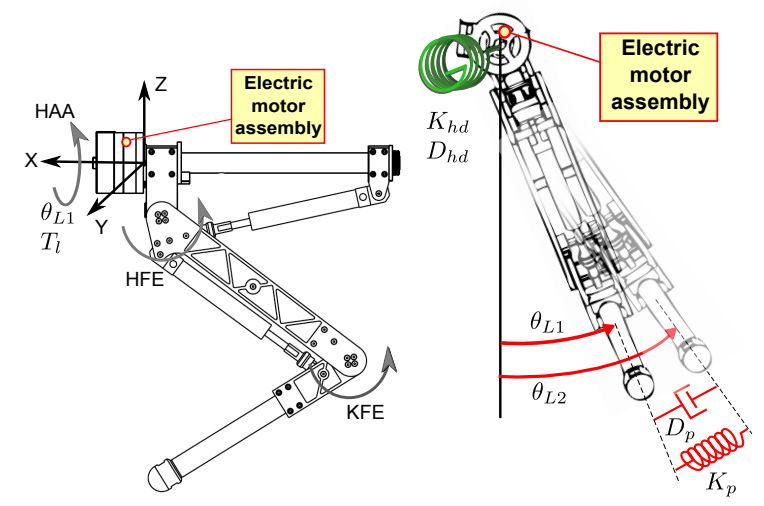

Fig. 2: HyQ Leg. Lateral (left) and frontal (right) view. The figures show the definition of the joints and their angles, as well as the coordinate frame.

Normally, this type of assembly is modelled by two second order differential equations coupled via the gearbox transmission flexibility [29]. However, after performing several open loop tests using chirp signals, an antiresonance was detected for the link velocity (see the frequency response of the link velocity to a chirp input voltage in Fig. 5).

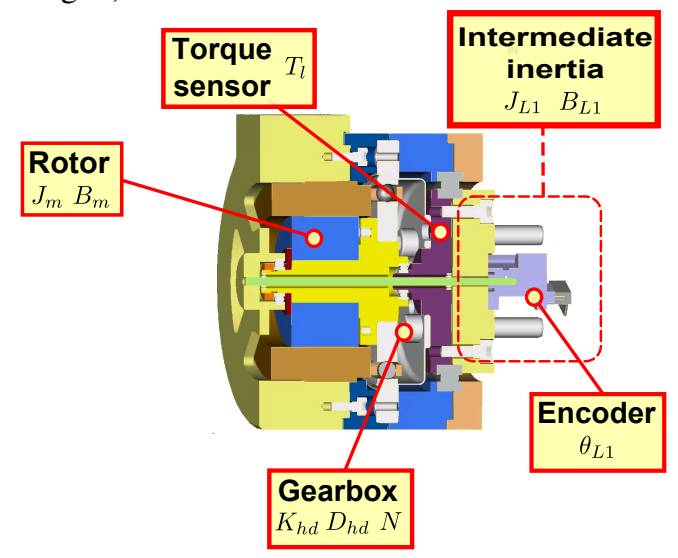

Fig. 3: Cross-section of the mechanical assembly of the electric joint. The intermediate inertia $J_{L 1}$ represents the part that interfaces the leg with the gearbox output.

Since a model with two inertia and one spring cannot capture this behavior, a more complex model with three inertia coupled by springs was used, as shown in the schematic in Fig. 4 where $K_{h d}$ and $K_{p}$ are the stiffness related to the gearbox and the leg flexibility, respectively. 


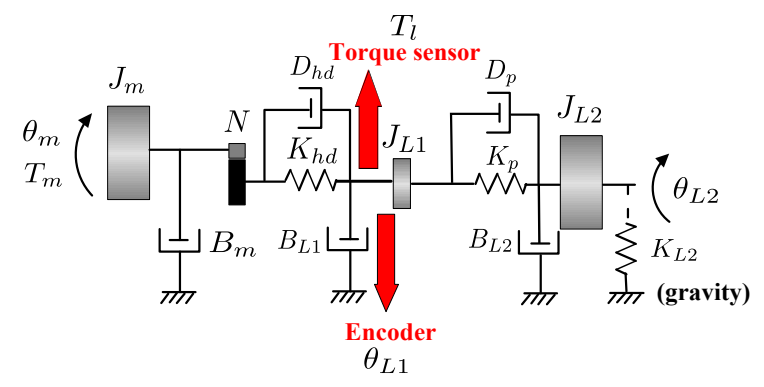

Fig. 4: Motor drive system with torsional load, schematic for the 3 mass- 2 spring model.

Joint position and torque are measured by an encoder and a torque sensor. Due to the topology depicted in Fig. 3 the position encoder measures the angle of the intermediate inertia $J_{L 1}$ while the link velocity is measured by averaging first order differences (4 samples) of the position encoder (averaging filter). In addition a strain gage based torque sensor is mounted at the output of the harmonic drive. No filter is implemented on the torque signal because it would introduce delays in the control action. According to this model, the Laplace transforms of the differential equations that describe the linearized dynamics of the load and of the electric motor are:

$$
\begin{aligned}
& I_{m}=\frac{V_{m}}{L s+R}-\frac{k_{w} s \theta_{m}}{L s+R} \\
& \theta_{m}=\frac{k_{t} I_{m}}{\left(J_{m} s+B_{m}\right) s}-\frac{K_{h d}+s D_{h d}}{N\left(J_{m} s+B_{m}\right) s}\left(\frac{\theta_{m}}{N}-\theta_{L 1}\right)+T_{f r} \\
& \theta_{L 1}=\frac{-\left(K_{p}+s D_{p}\right)}{\left(J_{L 1} s+B_{L 1}\right) s}\left(\theta_{L 1}-\theta_{L 2}\right)+\frac{K_{h d}+s D_{h d}}{\left(J_{L 1} s+B_{L 1}\right) s}\left(\frac{\theta_{m}}{N}-\theta_{L 1}\right) \\
& \theta_{L 2}=\frac{K_{p}+s D_{p}}{\left(J_{L 2} s+B_{L 2}\right) s}\left(\theta_{L 1}-\theta_{L 2}\right)-\frac{K_{L 2} \theta_{L 2}}{\left(J_{L 2} s+B_{L 2}\right) s}+T_{d i s t}
\end{aligned}
$$
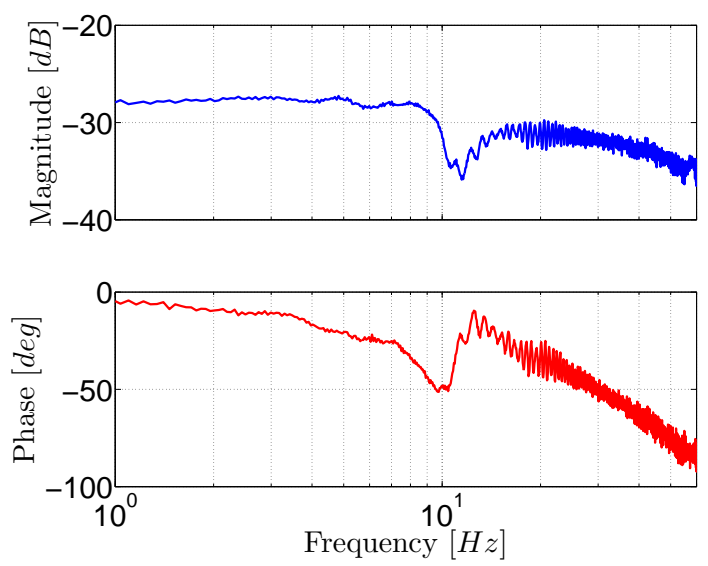

Fig. 5: Frequency response of the link velocity $\dot{\theta}_{L 1}$ to a chirp input voltage at the HAA motor, experimentally obtained with an unconstrained HyQ leg. where $I_{m}, \theta_{m}$ denote the motor current and motor position; $\theta_{L 1}$ and $\theta_{L 2}$ are the intermediate inertia and leg positions; $V_{m}$ is the motor voltage; $T_{f r}$ is the friction torque in the harmonic drive and $T_{d i s t}$ is an external disturbance torque applied to the leg. All other symbols and parameter values are given in Table 1. A block diagram that illustrates the relationships between the state variables is depicted in Fig. 6. Since the rotational inertia of the leg $J_{L 2}$ varies with the configuration of the joints HFE and KFE, the table includes also upper and lower bounds for the leg inertia. In particular the fact that the leg can retract or extend results in different mass distributions around the hip abductionadduction axis. Namely, the inertia is higher when the leg is extended and lower when it is retracted. Gravity has also an effect on the load dynamics, that, when linearized, behaves as a rotational spring $K_{L 2}$. The stiffness of this "gravity spring" is also dependent on the leg configuration and is reported in Table 1 for an extended and retracted leg configuration.

Table 1: Model parameters

\begin{tabular}{lll}
\hline \hline Name & Model Parameters & Value \\
\hline \hline$J_{m}$ & Rotor + gearbox inertia (D) & $5.72 \cdot 10^{-5} \mathrm{kgm}^{2}$ \\
\hline$K_{h d}$ & Gearbox stiffness (I) & $8.077 \cdot 10^{3} \mathrm{Nm} / \mathrm{rad}$ \\
\hline$D_{h d}$ & Gearbox damping (I) & $16.56 \mathrm{Nms} / \mathrm{rad}$ \\
\hline$B_{m}$ & Visc. frict. rotor (I) & $0.0015 \mathrm{Nms} / \mathrm{rad}$ \\
\hline$J_{L 1}$ & Interm. inertia (I) & $1 \cdot 10^{-4} \mathrm{kgm}^{2}$ \\
\hline$B_{L 1}$ & Visc. frict. of inertia $J_{L 1}$ (I) & $0 \mathrm{Nms} / \mathrm{rad}^{2}$ \\
\hline \multirow{2}{*}{$J_{L 2}$} & Leg inertia (C) & $0.439 \mathrm{kgm}^{2}(\mathrm{ext})$. \\
& & $0.129 \mathrm{kgm}^{2}$ (ret.) \\
\hline$B_{L 2}$ & Visc. frict. of inertia $J_{L 2}$ (I) & $0.756 \mathrm{Nms} / \mathrm{rad}$ \\
\hline \multirow{2}{*}{$K_{L 2}$} & Linear stiffness & $11.2 \mathrm{Nm} / \mathrm{rad}(\mathrm{ext})$. \\
& due to gravity & $7.17 \mathrm{Nm} / \mathrm{rad}(\mathrm{ret})$. \\
\hline$K_{p}$ & Leg stiffness (I) & $1.923 \cdot 10^{3} \mathrm{Nm} / \mathrm{rad}$ \\
\hline$D_{p}$ & Leg damping (I) & $7.56 \mathrm{Nms} / \mathrm{rad}$ \\
\hline$L$ & Coil inductance (D) & $2.02 \cdot 10^{-3} \mathrm{H}$ \\
\hline$R$ & Coil resistance (D) & $3.32 \Omega$ \\
\hline$k_{t}$ & Motor torque constant (D) & $0.19 \mathrm{Nm} / \mathrm{A}$ \\
\hline$k_{w}$ & Motor speed constant (D) & $0.19 \mathrm{Nms} / \mathrm{rad}$ \\
\hline$N$ & Gear ratio (D) & 100 \\
\hline \hline
\end{tabular}




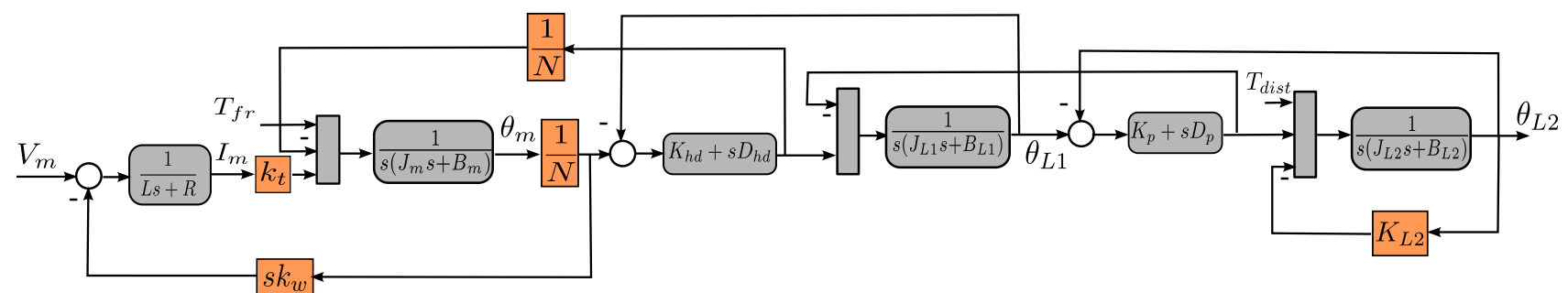

Fig. 6 Block diagram representing Eqn. (1) that describes the linearized dynamics of the electric motor and of the load. $V_{m}$ is the voltage input, $T_{f r}$ the harmonic-drive disturbance torque, $T_{d i s t}$ is an external disturbance torque coming from the load side, $\theta_{m}$ is the motor position, $\theta_{L 1}$ and $\theta_{L 2}$ the positions of the intermediate and leg inertia, respectively.

\begin{tabular}{lll} 
Name & State variables & Unit \\
\hline \hline$\theta_{m}$ & Ang. pos. of the rotor & $\mathrm{rad}$ \\
\hline$\theta_{L 1}$ & Ang. pos. of the intermediate inertia & $\mathrm{rad}$ \\
\hline$\theta_{L 2}$ & Ang. pos. of the leg & $\mathrm{rad}$ \\
\hline$I_{m}$ & Motor current & $\mathrm{A}$ \\
\hline \hline Name & Inputs & Unit \\
\hline \hline$V_{m}$ & Motor voltage & $V$ \\
\hline$V_{m_{V C}}$ & Vel. comp. voltage & $V$ \\
\hline$T_{d i s t}$ & Ext. dist. torque (load side) & $\mathrm{Nm}$ \\
\hline$T_{f r}$ & Frict. dist. torque (motor side) & $\mathrm{Nm}$ \\
\hline \hline Name & Outputs & Unit \\
\hline \hline$T_{l}$ & Load torque & $\mathrm{Nm}$ \\
\hline \hline Name & Controller Gains & \\
\hline \hline$P_{t}$ & Torque controller prop. gain & \\
\hline$I_{t}$ & Torque controller integral gain & \\
\hline$\beta$ & Gain of the PI torque controller & \\
\hline$\alpha$ & Velocity compensation (scalar) gain & \\
\hline \hline Name & Transfer functions \\
\hline \hline$P I_{t}(z)$ & PI torque controller \\
\hline$G_{t}(s)$ & TF between $V_{m}$ and $T_{l}$ & \\
\hline$V C_{g a i n}$ & TF of velocity compensation \\
\hline$G_{t_{V C}}(s)$ & TF between $V_{m}$ and $T_{l}$ after vel. comp. \\
\hline \hline
\end{tabular}

vary in a specified range. The inner torque loop and the velocity feedback loop must be designed to be consistent with these requirements.

\subsection{Positive feedback velocity compensation}

One difficulty in the design of the torque loop controller is that the load dynamics may introduce severe limitations in the closed loop performance of the torque loop. This problem has been largely overlooked since in many cases the load dynamics are ignored in the analysis. In this subsection a positive velocity feedback (velocity compensation) is introduced to address these limitations and improve the torque bandwidth. To exhibit the above-mentioned limitations, first of all, the system response has been considered after closing the inner velocity feedback loop. The torque transmitted to the load is measured by the torque sensor and can be expressed as:

$$
T_{l}=\left(K_{h d}+s D_{h d}\right)\left(\frac{\theta_{m}}{N}-\theta_{L 1}\right)
$$

From Eqn. (1), Eqn. (2) and Fig. 7, closing the velocity loop (without considering the averaging filter for the sake of simplicity), the transfer function from the motor voltage to the torque is given by (cf. Eq. 3.24, Ch. 4, p. 45 in [30] for complete derivation):

$$
G_{t_{V C}}=\frac{k_{t}\left(D_{h d} s+K_{h d}\right) p_{1}}{N\left(p_{1} q_{1}+\left(q_{2}-V C_{\text {gain }} s\right) q_{3}\right)}
$$

\section{Controller Design}

This section explains the design of the control system. The controller architecture is shown in Fig. 7 where an inner positive velocity feedback loop is followed by a torque loop controller and finally an outer impedance (position) loop. Specifications for the impedance loop vary depending on the gait, for example a trotting gait frequency is around $2 \mathrm{~Hz}$ for HyQ that has a mass of $75 \mathrm{~kg}$. The specifications for the performance will depend on the type of locomotion gait and the gains of the impedance loop will where:

$$
\begin{aligned}
p_{1}= & \left(J_{L 2} s^{2}+B_{L 2} s+K_{L 2}\right)\left(J_{L 1} s^{2}+B_{L 1} s\right) \\
& +\left[\left(J_{L 2} s^{2}+B_{L 2} s+K_{L 2}\right)+\left(J_{L 1} s^{2}+B_{L 1} s\right)\right]\left(D_{p} s+K_{p}\right) \\
q_{1}= & (L s+R)\left(J_{m} s^{2}+B_{m} s+\frac{\left(D_{h d} s+K_{h d}\right)}{N^{2}}\right)+k_{t} k_{w} s \\
q_{2}= & \frac{N}{k_{t}}(L s+R)\left(J_{m} s^{2}+B_{m} s\right)+N k_{w} s \\
q_{3}= & \frac{k_{t}}{N}\left(J_{L 2} s^{2}+B_{L 2} s+D_{p} s+K_{p}+K_{L 2}\right)\left(D_{h d} s+K_{h d}\right)
\end{aligned}
$$




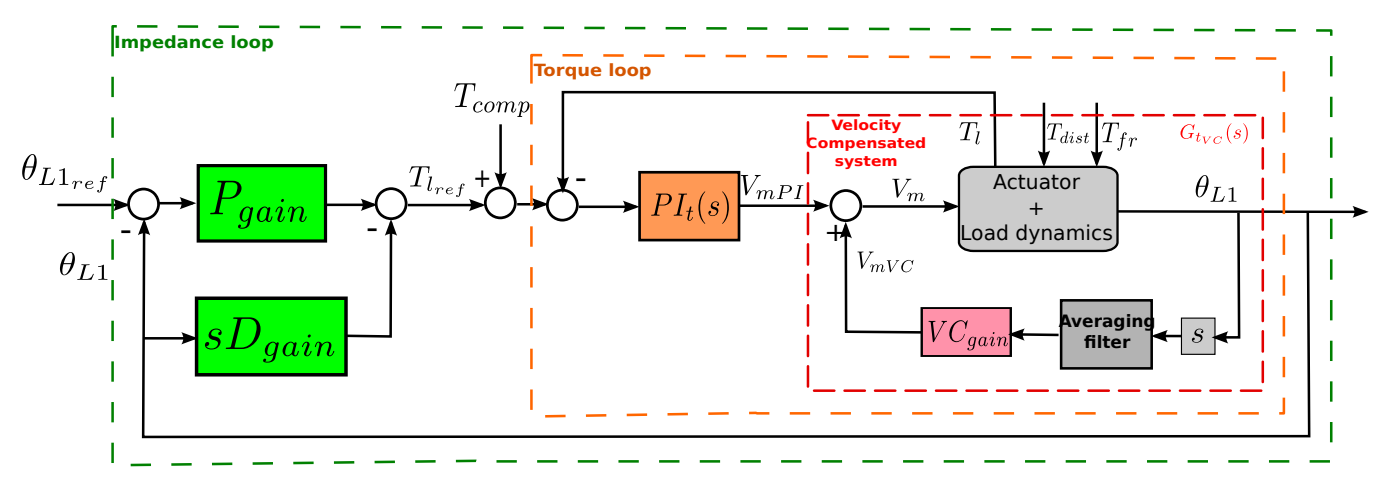

Fig. 7 Block diagram of the velocity compensated system with inner torque loop (PI, orange block) outer impedance loop (PD, green blocks). The velocity compensation term $\left(V_{m V C}\right)$ is added to the output $\left(V_{m P I}\right)$ of the torque controller.

Observe that the transmission zeros in Eqn. (3) introduced by the polynomial $p_{1}$ depend entirely on the load dynamics, that is the load connected at the output of the harmonic drive. When the damping coefficients $B_{L 1}$ and $B_{L 2}$ are small, as is usually the case, some of these transmission zeros are very close to the stability region boundary. In the case of continuous time systems the boundary is the imaginary axis and in the case of discrete time systems this is the unit circle. Notice that the zeros may be real or complex depending on the value of the stiffness $K_{L 2}$. These zeros impose limitations in the achievable closed loop bandwidth when using a simple proportional and integral torque controller. This is because the controller pole, located at the origin, will be attracted towards the transmission zeros becoming the dominant pole of the system, thus limiting the closed loop bandwidth of the torque loop unless very high gains are used in the torque controller. In most cases the torque loop gain will have a finite gain margin and therefore the controller gain cannot be made sufficiently large. This is more pronounced in digital control where the gain margin is likely to be much lower than the gain margin for a continuous time system. The effect of the velocity compensation is that these unwanted transmission zeros (polynomial $p_{1}$ ) can be cancelled if the velocity feedback gain is chosen as $V C_{\text {gain }}=q_{2} / s$ so that the term $q_{2}-V C_{\text {gain }} s$ in the denominator of Eq. (3) is equal to zero.

$$
V C_{\text {gain }}=\frac{N}{k_{t}}(L s+R)\left(J_{m} s+B_{m}\right)+N k_{w}
$$

The implementation of the compensator requires derivatives of the velocity signal that is often prone to quantization errors. Since this derivative is likely to be noisy the compensator has to be approximated by adding suitable filters which would add delay. An alternative solution is to use a simplified velocity compensation as presented in [31] which is obtained by discarding the derivative terms from Eq. (4):

$$
V C_{\text {gain }}=\frac{\alpha N}{k_{t}}\left(R B_{m}+k_{t} k_{w}\right) \quad \alpha>0
$$

This simplified velocity compensation is obtained by setting $L=0$ and $J_{m}=0$. This means we are neglecting both the electrical dynamics and the acceleration term, which would introduce noise in the system. $\alpha$ is introduced as an adjustable parameter. Therefore, with Eq. (5), an exact cancellation of the transmission zeros $p_{1}$ is generally not possible. Nevertheless, even though an exact cancellation is not possible, an improvement in the closed loop torque bandwidth can be achieved. To understand when and how this is possible, consider the velocity compensation given by Eq. (5). For the parameter values given in Table 1 and setting $K_{L 2}=0$, the polynomial $p_{1}$ has four real roots $z_{4}<z_{3}<z_{2}<z_{1}=0$. The transfer function (3) also has a pole at zero. This is an unobservable pole and therefore it cancels out with the zero $z_{1}$. The second zero $z_{2}$ is the closest to the imaginary axis and limits the torque bandwidth that can be achieved with a PI torque controller. Indeed, as $\alpha$ increases one real pole in Eq. (3) moves towards the stability boundary along the real axis and at some point it will become identical to $z_{2}$. The value of the gain $\alpha$ for which this happens, is the ideal value required for cancelling out unwanted zero $z_{2}$. In this particular case, $z_{2}$ varies as a function of the leg inertia. Hence, it may be difficult to completely cancel out this zero for all leg configurations with a fixed value for $\alpha$. However, as long as the pole in Eq. (3) is placed to the right of the zero $z_{2}$ then the bandwidth limitation introduced by this zero is avoided (cf. Sec. 3.5.3, Ch. 4, p. 46 in [30]). When $K_{L 2} \neq 0$ then the roots of $p_{1}$, which are closest to the imaginary axis, are complex ( $z_{1}$ and $z_{2}$ are complex conjugate). As $\alpha$ increases two poles in Eq. (3) will move towards the imaginary axis as a complex conjugate pair but there is no value of $\alpha$ that will completely cancel out the unwanted zeros $z_{1}$ and $z_{2}$. In this case the velocity compensation will not be as effective 
as for the case where $K_{L 2}=0$. Nevertheless, it still results in an improvement of the achievable closed loop torque bandwidth as shown in Fig. 8.
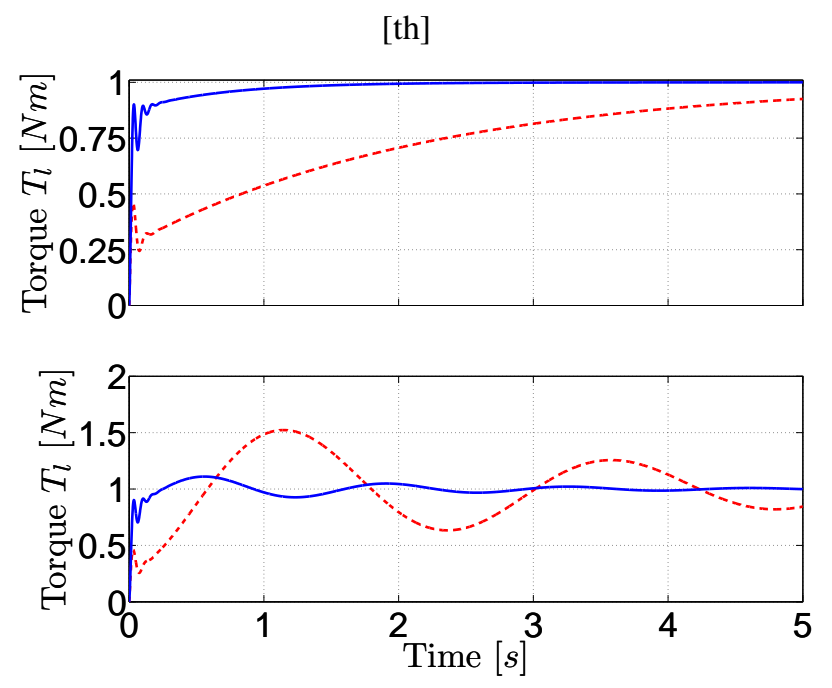

Fig. 8: Simulation. Unit torque step responses with $K_{L 2}=0$ (upper plot) and $K_{L 2}=11.2 \mathrm{Nm} / \mathrm{rad}$ (lower plot) for different velocity compensation gains $\alpha=0.94$ (Solid line) and $\alpha=0$ (dashed line). An extended leg inertia $\left(J_{L 2}=0.439\right.$ $\mathrm{kgm}^{2}$ ) has been considered. The use of velocity compensation significantly reduces the response time.

Similar results can be established after discretization of the continuous time system. A final remark is that an alternative to overcome the limitations imposed by the unwanted zeros is to consider more complex controllers, for example a double integrator PII controller, or designs based on loop shaping control which introduce additional poles and zeros. The disadvantage is that tuning these complex controllers cannot be done without a good model for the system.

\subsection{Torque controller design}

In this section the design of a PI torque controller is considered. An integrator is included in the controller structure to remove steady state errors when tracking constant torque inputs or when constant disturbances arise. The integrator is implemented in discrete time using the backward euler approximation. This controller is a lag compensator that has one zero and one pole. The analysis and design are carried out in discrete time because sampling introduces noticeable differences in comparison to the continuous time case. For example the system can become non-minimum phase even if the underlying continuous time is of minimum phase (non-minimum phase systems are more difficult to control). The equation of the PI controller is given by:

$$
P I_{t}(z)=P_{t}+I_{t} \frac{z T_{s}}{z-1}=\left(P_{t}+I_{t} T_{s}\right) \frac{\left(z-\frac{P_{t}}{P_{t}+I_{t} T_{s}}\right)}{z-1}
$$

where $z$ is the $Z$-transform variable and $T_{s}=1 \mathrm{~ms}$ is the sampling time interval; $P_{t}$ and $I_{t}$ are the proportional and integral gains to be determined. We remark that small sampling time intervals, on one hand, improve the disturbance rejection properties of the closed loop system. However, as the sampling time interval decreases, the effects of quantization noise in the encoders become more prominent, especially when computing velocities from position measurements using simple first order differences. In addition, small sampling time intervals can introduce nonminimum phase behavior in the sampled system which is more difficult to control. The selected sampling time of 1 $m s$ is a trade-off among all these aspects. At the actual encoder resolution ( 80000 count/rev), the smallest velocity that can be measured with $1 \mathrm{~ms}$ sampling time interval, is $0.0125 \mathrm{rad} / \mathrm{s}$.

Traditionally, the design of an inner loop controller is carried out with the aim of maximizing the closed loop bandwidth of the inner loop. However, one of the first difficulties is how to measure the bandwidth of the torque loop. The closed loop torque bandwidth for an unconstrained system, for example when the leg is moving freely in the air, is very different from the case when the system is in contact with the ground and depends on the ground stiffness (soft versus hard). In fact, assuming that gravity is fully compensated (this means $K_{L 2}=0$ ), the free leg motion with the torque loop closed is not internally stable (as was shown in the previous section there is a pole-zero cancellation on the stability boundary). Further, it is not obvious that maximizing the bandwidth of the torque loop is always consistent with the specifications for the outer impedance loop. In the approach presented in this paper it was decided to design the controller gains so that the torque loop gain has a phase margin larger than $30^{\circ}$ and a gain margin larger than $12 d B$ for the upper and lower bounds of $J_{L 2}$ and $K_{L 2}$ and with a velocity compensation gain $\alpha=0.94$. This would result in a satisfactory response. In addition, the closed loop torque response was required to be stable for all values of the velocity compensation gain $\alpha$ between zero and one (but the gain and phase margins can be less than $12 \mathrm{~dB}$ or 30 degrees, respectively). A set of controller gains satisfying the given specifications is found 
by using the Matlab SISOtool.

$$
P_{t}=0.382 \beta \quad I_{t}=18 \beta \quad 25>\beta>0
$$

Changing the gain $\beta$ only affects the gain of the controller but the controller zero remains fixed. This gives ample freedom to investigate the effects of increasing the torque loop gain $\beta$ and hence increasing the closed loop torque bandwidth when the outer loop specifications are considered. We must remark that, when defining the torque bandwidth, we consider that the system is free to move in the air. Then the bandwidth is the frequency where the torque amplitude decreases by $-3 d B$ respect to the reference.

\subsubsection{Harmonic drive torque ripple compensation}

One drawback of using a harmonic drive gearbox is that it introduces torque ripples $\left(T_{f r}\right.$ in (1)). The problem is related to the working principle of the gearbox that is based on the motion of an elliptic element (wave generator). This motion creates torque fluctuation with a fundamental frequency which is twice the wave generator angular velocity. While this disturbance is normally neglected in position control schemes, because it is passively filtered out by the inertia of the system, conversely it has a detrimental effect on torque control and creates vibrations and wearing of the components. A way to mitigate this problem is to add a lead/lag compensator (notch) in series to the PI controller in order to add enough phase lead at the resonance of the transfer function between $T_{f r}$ and $T_{l}$ where the ripple is more prominent, as illustrated in Sec. 3.7, Ch. 4, p. 50 in [30].

\subsection{Impedance control}

An impedance controller is added as an outer loop as shown in Fig. 7. The controller gains $P_{\text {gain }}$ and $D_{\text {gain }}$ represent the stiffness and damping for the joint. The output $T_{l_{r e f}}$ of the controller provides the reference torque for the inner loop:

$$
T_{l_{\text {ref }}}=P_{\text {gain }}\left(\theta_{L 1_{\text {ref }}}-\theta_{L 1}\right)-D_{\text {gain }} \dot{\theta}_{L 1}
$$

while $\theta_{L 1_{r e f}}$ is the desired trajectory for the joint position. The term involving the link velocity feedback is implemented using an averaging filter to reduce the effects of encoder quantization. $T_{I D}$ (see Fig. 7) is an external compensation torque (e.g. inverse dynamics) that can be added to remove the effects of gravity and inertia (and thus reduce position tracking errors):

$$
T_{I D}=\left(J_{L 1}+J_{L 2}\right) \ddot{\theta}_{L 2_{r e f}}+m g l_{c o m} \sin \left(\theta_{L 2}\right)
$$

where $l_{\text {com }}$ is the distance of the leg center of mass from the joint axis. For HyQ, a range of values for the impedance loop gains that is considered to be sufficient for walking, trotting and running tasks is $P_{\text {gain }} \in[1,2000] \mathrm{Nm} / \mathrm{rad}$ and $D_{\text {gain }} \in[1,50] \mathrm{Nms} / \mathrm{rad}$.

\section{Stability regions}

The analysis here is limited to the abduction-adduction electric joint of HyQ with a variable load inertia that depends on the configuration of the leg joints. In particular, given a range of impedance parameters, $P_{\text {gain }}$ and $D_{\text {gain }}$, this analysis will assess how the region of closed loop stability is affected by varying the torque controller gain $(\beta)$, the velocity compensation gain $(\alpha)$, the number of samples used in the averaging filter $\left(N_{a v}\right)$ and the sampling time $\left(T_{s}\right)$.

The analysis has been performed by varying the stiffness $P_{\text {gain }}$ between 1 and $20000 \mathrm{Nm} / \mathrm{rad}$ and the damping $D_{\text {gain }}$ between 1 and 50. The upper-bound for the stiffness was chosen such that we could determine the boundary for the stability and passivity regions and is way beyond the maximum value used in locomotion. The stability of the overall system is determined by computing the closed loop eigenvalues and checking that they are inside the unit circle. In addition, when closed loop stability is attained, the region where the phase margin is less than 30 degrees can be determined. These calculations were carried out in Matlab using the mathematical model presented in Section 2. The results are displayed in Figs. 9, 10, 11 and 12 where the white area corresponds to the stable region; light grey is a stable region with a phase margin of less than 30 degrees and the dark area is the unstable region. In the analysis all the regions have been computed for the leg in stretched configuration $J_{L 2}=0.439 \mathrm{kgm}^{2}$ unless it is otherwise stated. Fig. 9 shows that as the torque controller gain increases, the unstable region for low stiffness and damping decreases but the unstable region for large stiffness and/or large damping increases. This clearly illustrates that increasing the torque loop bandwidth may not be consistent with the (stability) requirements for the outer impedance loop. This can be explained if we consider that for any given system and controller architecture, there is a limit on the maximum loop gain thatcan be achieved, beyond which stability is not ensured and performance degrades. In a nested architecture both loops contribute to the loop gain. If the loop gain contribution from the torque loop increases (e.g. the gain $\beta$ increases), then the contribution from the impedance loop should reduce otherwise 


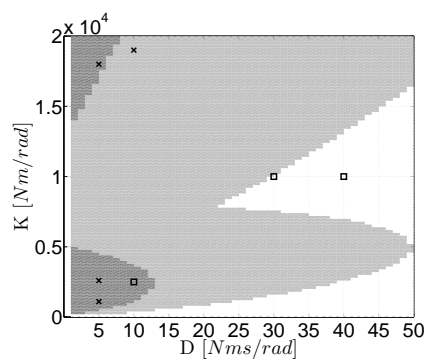

(a) $\beta=1$

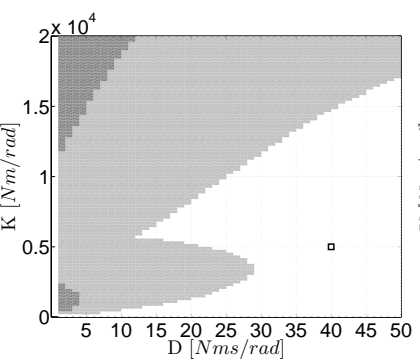

(b) $\beta=2$

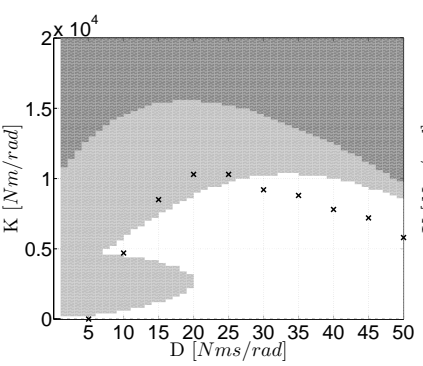

(c) $\beta=4$

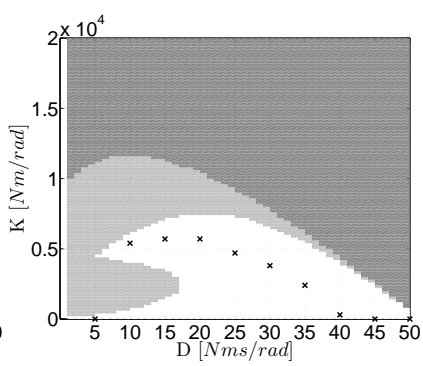

(d) $\beta=6$

Fig. 9 Stability regions varying the torque controller gain $\beta$ (with $\alpha=0.94, N_{a v}=4$ and $T_{s}=1 \mathrm{~ms}$ ). White area corresponds to the stable region; light grey is a stable region with a phase margin of less than 30 degrees and the dark area is the unstable region. Crosses and squares denote unstable and stable experimental points respectively.

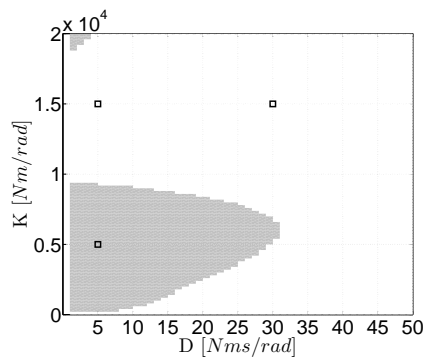

(a) $\alpha=0$

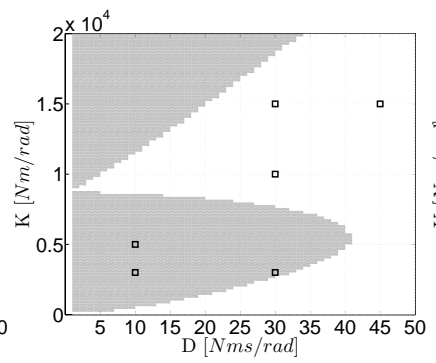

(b) $\alpha=0.5$



(c) $\alpha=0.94$

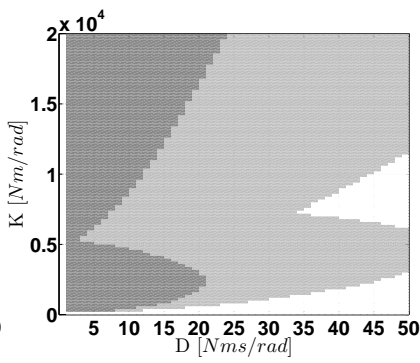

(d) $\alpha=1.2$

Fig. 10 Stability regions varying the velocity compensation gain $\alpha$ (with $\beta=1, N_{a v}=4$ and $T_{s}=1 \mathrm{~ms}$ ). White area corresponds to the stable region; light grey is a stable region with a phase margin of less than 30 degrees and the dark area is the unstable region. Crosses and squares denote unstable and stable experimental points respectively.

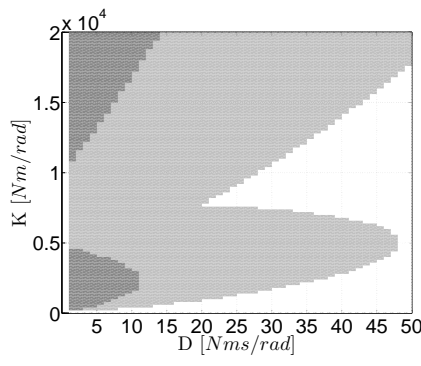

(a) $N_{a v}=1$

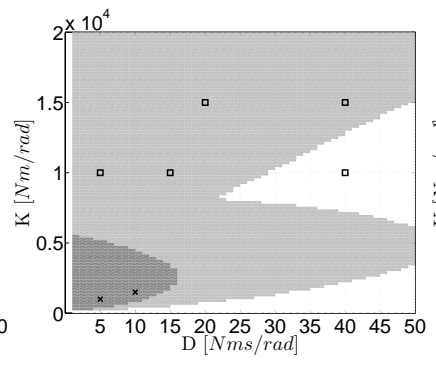

(b) $N_{a v}=10$

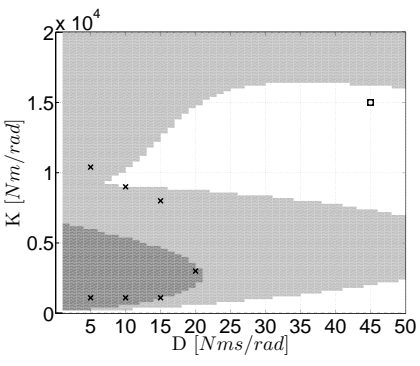

(c) $N_{a v}=20$



(d) $N_{a v}=50$

Fig. 11 Stability regions varying number of samples $N_{a v}$ of the link velocity filter (with $\beta=1, \alpha=0.94$ and $T_{s}=1 \mathrm{~ms}$ ). White area corresponds to the stable region; light grey is a stable region with a phase margin of less than 30 degrees and the dark area is the unstable region. Crosses and squares denote unstable and stable experimental points respectively.

closed loop stability would be lost. From Figs. 10 (a), (b) and (c) it is clear that increasing the velocity compensation gain results in an increasing instability region. Once more this gives further evidence that as the torque loop bandwidth increases, the outer loop may become unstable. The effects of increasing the number of samples $\left(N_{a v}\right)$ in the velocity averaging filter are shown in Fig. 11. Averaging a large number of samples enlarges the instability region for low stiffness values but the unstable region for high stiffness and low damping decreases in size. Figure 12 clearly shows that for a large sampling time interval the region for instability is the largest both for low and high damping and stiffness. The region where the phase margin is less than 30 degrees also increases as the sampling time increases. For low leg inertia configuration in Fig. 12 (d) the instability region for low damping and low stiffness increases but the unstable region decreases for low damping and large stiffness. The region with a phase margin smaller than 30 degrees is also larger for the low inertia configuration.

To determine the stability regions experimentally is not 


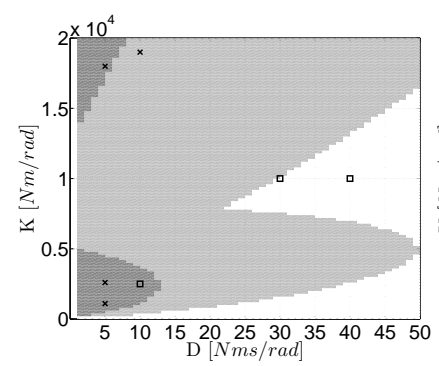

(a) $T_{s}=1 \mathrm{~ms}$

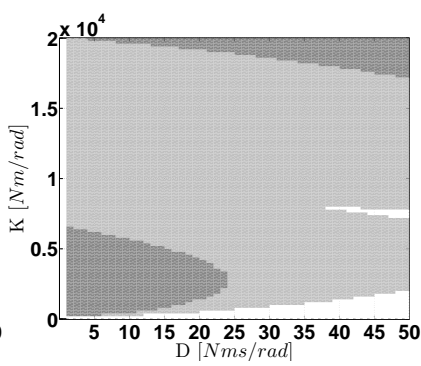

(b) $T_{s}=6 \mathrm{~ms}$

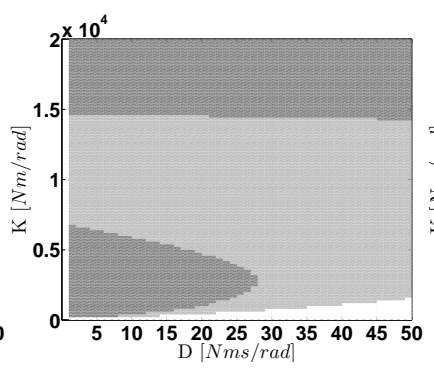

(c) $T_{s}=8 \mathrm{~ms}$

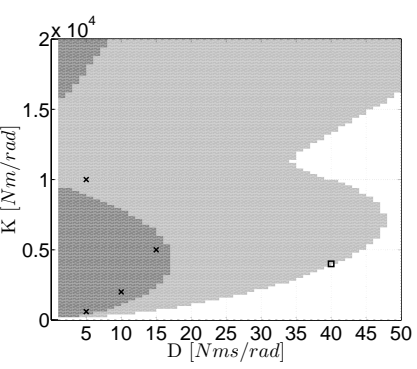

(d) $J_{L 2}=0.129 \mathrm{kgm}^{2}$

Fig. 12 (a),(b),(c) Stability regions varying the sampling times $T_{s}$ (with $\beta=1, N_{a v}=4, \alpha=0.94$ ). (d) Stability region for retracted leg (lower inertia) $J_{L 2}=0.129 \mathrm{kgm}^{2}$ (with $\beta=1, \alpha=0.94, N_{a v}=4$ and $T_{s}=1 \mathrm{~ms}$ ). White area corresponds to the stable region; light grey is a stable region with a phase margin of less than 30 degrees and the dark area is the unstable region. Crosses and squares denote unstable and stable experimental points respectively.

an easy task because it would involve a vey large number of experiments. Even only finding the stability boundaries is not a simple task. Several experimental tests were carried out to validate the analytical results predicted by the model. The adopted methodology was to start from values $P_{\text {gain }}$ and $D_{\text {gain }}$ well inside the stable region and change the parameters in small steps until the instability was triggered. This enabled us to get a rough idea of the boundary of the stability region. The experimental results are displayed in Fig. 9, 10, 11 and 12, where crosses and squares denote unstable and stable points respectively. With the exception of Fig. 9(c) and 9 (d), overall the experimental tests are consistent with the analytical calculations. Fig. 9 (b), 10 (a), 10 (b) and 11 (b) are fully in agreement with the theoretical results. For test points near the stability boundaries inconsistencies are present suggesting that the model lacks accuracy from a quantitative point of view but qualitatively it is correct. The experimental results shown in Fig. 9 (c) and 9 (d) are quite different from the expected outcome. Even though a precise explanation is currently not available, the authors have several possible hypothesis for these discrepancies: the experimental set-up might have reached some limiting conditions, which invalidate the linear analysis (for example by generating motor voltages that exceed the capabilities of the motor drive electronics, or by generating reference torques that exceed the range of the torque sensor). Their investigation is part of future work.

\section{Passivity analysis}

Another question of interest is whether the closed loop system remains stable when it interacts with a passive environment. It is well-known that a strictly passive system, connected to any passive environment, is necessarily stable [32]. Thus, since most terrain surfaces are passive, to ensure a stable contact with the environment also the robot joints have to be passive. This section therefore analyzes the main factors that influence passivity. Stating that a system is passive, is equivalent to saying that the system is intrinsically dissipative. This is not always the case when compliance is obtained actively, where the compliant behavior is emulated by controlled actuators. In this case, the controller gains can destroy passivity. The requirement to ensure this type of stability for the robot interacting with the environment is the following: the port of interaction between the system and the environment, i.e. the driving port impedance, has to be passive. For linear time invariant systems this is a necessary and sufficient condition, but it is only a sufficient condition for nonlinear systems. Let $Z(s)$ denote the driving port impedance transfer function. Then $Z(s)$ is passive if and only if it is positive real [33]. In [16] and [34] it has been shown that this is equivalent to:

1. $Z(s)$ has no poles in $\Re(s)>0$;

2. the phase of $Z(s)$ lies between -90 and 90 degrees.

For sampled data control systems, Colgate [16] has suggested an approximate method based on computing the corresponding discrete time transfer function $Z(z)$, assuming that the port of interaction is also sampled. The phase of $Z(z)$ is computed and corrected by subtracting $\omega T_{s} / 2$ $\mathrm{rad}$, where $T_{s}$ is the sampling time interval. Although many studies have been carried out for analyzing the passivity of sampled-data systems [35], there is still a lack of information about the influence of the closed-loop torque control bandwidth on the combinations of stiffness and damping that can be passively rendered (in the field of haptics called Z-width) [16]. Therefore, this section will show that the torque loop performance plays an important role in determining the range of passively achievable impedance values. The discrete time transfer function $Z(z)$ (impedance) has been computed from the link velocity $\dot{\theta}_{L 1}$ to the load 
disturbance $T_{\text {dist }}$. The transfer function has been computed for two cases: one for the torque loop (with velocity compensation) and one for the system after closing the outer impedance loop. Then the above-mentioned phase correction is applied to include the fact that the system is sampled. In the approach presented in this paper the analysis of passivity has been done by varying several parameters to have a better understanding of their influence: the gain of the PI torque controller (by varying the gain $\beta$ in Eq. (7)), the velocity compensation gain $\alpha$, the sampling time $T_{s}$, and the number of samples $N_{a v}$ of the averaging filter of the link velocity. For each set of parameters the analysis was performed by first checking the stability of $Z(z)$ and then verifying that the corrected phase of $Z(z)$ was in the range $\pm 90^{\circ}$ for frequencies up to the Nyquist frequency. If these conditions are not satisfied then $Z(z)$ is not passive for the particular set of parameter values. The results of this analysis are summarized in Table 2.

Table 2: Passivity

\begin{tabular}{lccc}
\hline \hline & $\begin{array}{c}\text { Torque } \\
\text { loop }\end{array}$ & $\begin{array}{c}\text { Imp. loop } \\
P_{\text {gain }}=200 \\
D_{\text {gain }}=10\end{array}$ & $\begin{array}{c}\text { Imp. loop } \\
P=20000 \\
D=50\end{array}$ \\
\hline$\beta=1$ & No & Yes & Yes \\
\hline$\beta=0.5$ & No & Yes & No \\
\hline$\beta=2$ & No & Yes & No \\
\hline$\beta=4$ & No & Yes & Unstable \\
\hline$\beta=6$ & No & Yes & Unstable \\
\hline$\alpha=0$ & Yes & Yes & Yes \\
\hline$\alpha=0.5$ & Yes & Yes & Yes \\
\hline$T_{s}=4 \cdot 10^{-3}[s]$ & No & Yes & No \\
\hline$T_{s}=2 \cdot 10^{-3}[s]$ & No & Yes & No \\
\hline$T_{s}=0.5 \cdot 10^{-3}[s]$ & No & Yes & Yes \\
\hline Averag. $N_{a v}=1$ & No & Yes & Yes \\
\hline Averag. $N_{a v}=10$ & No & Yes & Yes \\
\hline Averag. $N_{a v}=20$ & No & Yes & Yes \\
\hline Averag. $N_{a v}=50$ & No & Yes & Yes \\
\hline $\begin{array}{l}\text { Low leg Inertia } \\
\text { (ret.) } J_{L 2}=0.129\end{array}$ & No & Yes & Yes \\
kgm ${ }^{2}$ & & & \\
\hline \hline
\end{tabular}

In this table the nominal set of parameters are: $\alpha=0.94$, $N_{a v}=4$ samples, leg inertia $J_{L 2}=0.439 \mathrm{kgm}^{2}, P_{t}=0.38$ and $I_{t}=18$. The overall (impedance + torque loop) system is always passive for low impedances $P_{\text {gain }}=200 \quad D_{\text {gain }}=$ 10 while passivity might be destroyed when the torque controller gain $\beta$ (and so the torque bandwidth) increases or the sampling frequency decreases. In particular it can be noticed that when the torque gain is larger than or equal to 4 the closed loop system with the impedance loop becomes unstable. This is a clear indication that increasing the bandwidth of the torque loop is not always consistent with the requirements of the position loop. When only the torque loop is closed the system is almost never passive except for low values of $\alpha$. The table shows that the velocity compensation is the key parameter affecting the passivity if the torque loop alone is considered. In particular when the amount of velocity compensation increases, the inner torque loop becomes not passive and therefore the torque control system alone can become unstable when the leg interacts with some environments. Further analysis showed that the system (without closing the impedance loop) becomes unstable if the leg is in contact with an environment with a stiffness $K_{L 2}$ between $72 \mathrm{Nm} / \mathrm{rad}$ and 3500 $\mathrm{Nm} / \mathrm{rad}$. This has been verified with experimental tests using the test setup depicted in Fig. 13 by commanding a leg motion to have an impact against a physical spring. This spring is positioned in order to create a certain stiffness $K_{L 2}$.

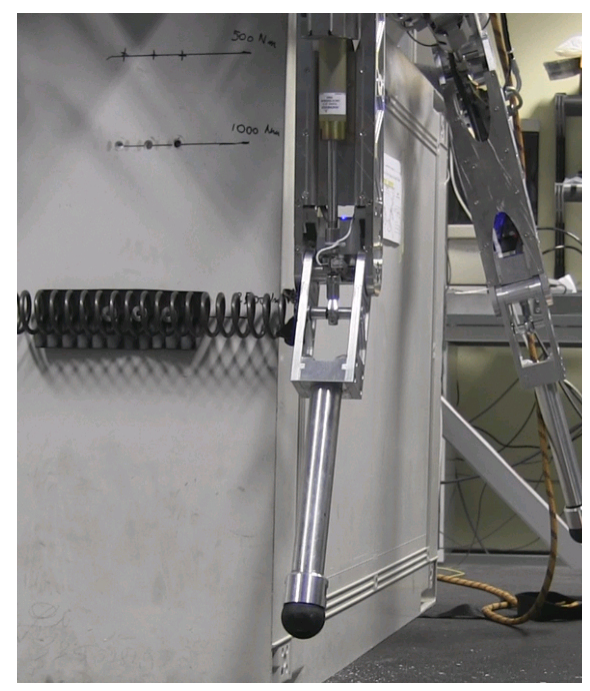

Fig. 13: Experimental setup for passivity tests. The rotational stiffness $K_{L 2}$ is obtained by positioning a linear spring at a certain distance from the $H A A$ axis.

Figure 14 shows the phase of $Z(z)$ when only the torque loop is closed ( $\beta=1$ and $\alpha=0.94$ ) and when both the torque and the impedance loops are closed $\left(P_{\text {gain }}=200\right.$, $D_{\text {gain }}=10$ ). The curves illustrate that, in the case of the torque loop alone, the phase of $Z(z)$ exceeds 90 degrees between 10 and $50 \mathrm{rad} / \mathrm{s}$, indicating the loss of passivity, while, when the outer loop is closed, the phase always remains within \pm 90 degrees, demonstrating that the passivity property is ensured as indicated in Table 2. 


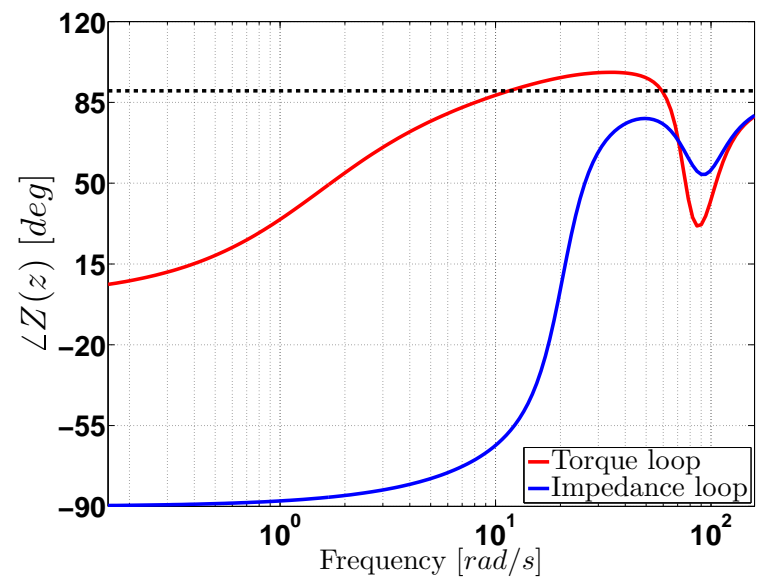

Fig. 14: Simulation. Phase plot of the driving port impedance $Z(z)$ when only the torque loop is closed $(\beta=1$ and $\alpha=0.94$ ) (red plot) and when both the torque and the impedance loops are closed $\left(P_{\text {gain }}=200, D_{\text {gain }}=10\right)$ (blue plot). The black line shows the limit of 90 degrees.

\section{Conclusions and future work}

This paper presented a methodology for designing joint impedance controllers based on an inner torque loop and a positive velocity feedback loop. In particular, it was shown that the positive velocity feedback can be used to increase the closed loop bandwidth of the torque loop without the need of a complex controller. It has been demonstrated that besides the sampling frequency and filtering, the bandwidth of the torque loop has a strong influence on the range of impedance parameters ( $Z$-width) that exhibit a passive and/or stable behavior. Indeed, larger inner loop bandwidth can be beneficial for disturbance rejection and to improve the tracking of the impedance (enlarge the range of frequencies for which the desired impedance is emulated by the system) but, at the same time, it can reduce the region of stable impedance parameters. This fact can limit the performance and versatility of a robot. Thus, the highest possible bandwidth for the torque loop might not be the best solution for all situations. It is therefore important to find a balance between the torque loop requirements (e.g. to have a good torque tracking) and the stability/passivity specifications of the overall system. Furthermore, it has become evident that, even for simple controllers, the design problem is challenging and that there are competing trade-offs to consider when selecting the controller gains.

This suggests that there is a need for a problem formulation that can encompass the design objectives in a more systematic and generic framework. There are a num- ber of areas that need further research and are left for future work. Adaptive schemes (e.g. gain scheduling) can be developed to modify the torque controller gains to satisfy the constraints of stability/passivity given by the desired impedance parameters specified by the system designer. The torque gains can also be modified depending on the load inertia variations that is changing with the leg configuration. In addition, varying the location of the PI torque controller zero can provide improvements in performance. The torque controller architecture can also be enhanced to reduce the effects of torque ripples arising in the gear transmission system (drive jitter). Since increasing the torque controller gain has been shown to be detrimental, this option for mitigating the drive jitter can be discarded and more different solutions must be found. Finally, there is a need to develop strategies to quantify the range of impedances that are required for specific tasks. At present there are some guidelines that only provide qualitative results, for example a high stiffness $\left(P_{\text {gain }}\right)$ is specified when there is contact with a compliant environment and the positioning accuracy is important. On the other hand, a low stiffness is used to maintain small contact forces or when the environment is stiff. Similarly, large damping values $\left(D_{\text {gain }}\right)$ are needed to reduce vibrations or to quickly dissipate energy. Future work also encompasses an extension of the methodology presented in this paper to include multidegree of freedom systems.

\section{Acknowledgements}

This research has been funded by the Fondazione Istituto Italiano di Tecnologia. J. Buchli is supported by a Swiss National Science Foundation professorship.

\section{References}

[1] Pratt, G. Low Impedance Walking Robots. Integr. Comp. Biol. 2002. pp. 174-181.

[2] Buchli, J., Kalakrishnan, M., Mistry, M., Pastor, P., and Schaal, S. Compliant quadruped locomotion over rough terrain. IEEE/RSJ International Conference on Intelligent Robots and Systems, 2009, pp. 814-820.

[3] Tsagarakis, N., Morfey, S., Medrano-Cerda, G., Li, Z., and Caldwell, D. G. COMAN a Compliant Humanoid: Optimal Joint Stiffness Tuning for Modal Frequency Control. IEEE International Conference on Robotics and Automation, 2013.

[4] Paine, N., Oh, S., and Sentis, L. Design and Control Considerations for High-Performance Series Elastic Actuators. IEEE/ASME Transactions on Mechatronics, 2014, pp. 1080 - 1091.

[5] Albu-Schäffer, A., Haddadin, S., Ott, C., Stemmer, A., Wimböck, T., and Hirzinger, G. The DLR lightweight robot: design and control 
concepts for robots in human environments. Industrial Robot: An International Journal. 2007. pp. 376-385.

[6] Ferris, D. P., Louie, M., and Farley, C.T. Running in the real world: adjusting leg stiffness for different surfaces.Proc. R. Soc. Lond. B., 1998, pp. 989-994.

[7] Hogan, N. Impedance control: An approach to manipulation: Part \{II $\}$ - Implementation. Journal of Dynamic Systems, Measurement, and Control,1985, pp. 8-16.

[8] Khatib, O. A unified approach for motion and force control of robot manipulators: The operational space formulation. Journal of Robotics and Automation. 1987, pp. 43-53.

[9] Raibert, M. H., and Craig, J. J., Hybrid position/force control of manipulators. Journal of Dynamic Systems, Measurement, and Control, 1981, pp. 126-133.

[10] Pratt, J., Chew, C., Torres, A., Dilworth, P., and Pratt, G. Virtual model control: An intuitive approach for bipedal locomotion. The International Journal of Robotics Research, 2001, pp. 129-143.

[11] Bosworth, W., Kim, S., and Hogan, N. The effect of leg impedance on stability and efficiency in quadrupedal trotting. IEEE/RSJ International Conference on Intelligent Robots and Systems, 2014, pp. 4895-4900.

[12] Pratt, G., and Williamson, M. Series elastic actuators. IEEE/RSJ International Conference on Intelligent Robots and Systems, 1995.

[13] Boaventura, T., Semini, C., Buchli, J., Frigerio, M., Focchi, M., and Caldwell, D. G., Dynamic torque control of a hydraulic quadruped robot. International Conference on Robotics and Automation, 2012, pp. 1889-1894.

[14] Hutter, M., Hoepflinger, M., and Gehring, C. Hybrid Operational Space Control for Compliant Legged Systems. In Proceedings of Robotics: Science and Systems, 2012.

[15] Mehling, J., Colgate, J., and Peshkin, M. Increasing the Impedance Range of a Haptic Display by Adding Electrical Damping. First Joint Eurohaptics Conference and Symposium on Haptic Interfaces for Virtual Environment and Teleoperator Systems, 2005, pp. 257262.

[16] Colgate, J. E. Coupled Stability of Multiport Systems - Theory and Experiments. Journal of Dynamic Systems, Measurement and Control,1994, 116, pp. 419-428.

[17] Ellis, G. Control System Design Guide. 2nd edition, Academic Press, London, 2000.

[18] Janabi-Sharifi, F., Hayward, V., and Chen, C.-S. Discrete-time adaptive windowing for velocity estimation. IEEE Transactions on Control Systems Technology,2000, pp. 1003-1009.

[19] Albu-Schaffer, a., Ott, C., and Hirzinger, G. A Unified Passivitybased Control Framework for Position, Torque and Impedance Control of Flexible Joint Robots. The International Journal of Robotics Research, 2007, pp. 23-39.

[20] Albu-Schaffer, A., Ott, C., and Hirzinger, G. A passivity based Cartesian impedance controller for flexible joint robots - part II: full state feedback, impedance design and experiments. International Conference on Robotics and Automation, 2004, Vol. 3, pp. 26662672.

[21] Hogan, N., and Colgate, E. Stability problems in contact tasks. In The robotics review 1, 2004, MIT Press, pp. 339-348.
[22] Karerooni, H., Sheridan, T. B., and Houpt, P. K. Robust compliant motion for manipulators, Part I: The fundamental concepts of compliant motion, Part II: Design method. Journal of Robotics and automation, 1986, pp. 83-105.

[23] Lawrence, D. A. Impedance control stability properties in common implementations. International Conference on Robotics and Automation, 1988, pp. 1185-1190.

[24] Buerger, S. P., and Hogan, N. Complementary Stability and Loop Shaping for Improved Human-Robot Interaction. Transactions on Robotics, 2007, pp. 232-244.

[25] Mehdi, H., and Boubaker, O. Stiffness and Impedance Control Using Lyapunov Theory for Robot-Aided Rehabilitation International Journal of Social Robotics, 2011, pp. 107-119.

[26] Yasrebi, N., and Constantinescu, D. Extending the z-width of a haptic device using acceleration feedback Haptics: Perception, Devices and Scenarios, 2008,Springer, pp. 157-162.

[27] Semini, C., Tsagarakis, N. G., Guglielmino, E., Focchi, M., Cannella, F., and Caldwell, D. G. Design of $\{\mathrm{HyQ}\}-$ a hydraulically and electrically actuated quadruped robot. Journal of Systems and Control Engineering, 2011.

[28] Focchi, M., Boaventura, T., Semini, C., Frigerio, M., Buchli, J., and Caldwell, D. G. Torque-control Based Compliant Actuation of a Quadruped Robot. In Proc. of the 12th IEEE Int. Workshop on Advanced Motion Control (AMC), 2012.

[29] Hori, Y., Iseki, H., and Sugiura, K. Basic consideration of vibration suppression and disturbance rejection control of multi-inertia system using SFLAC (state feedback and load acceleration control). Transactions on Industry Applications, 1994, pp. 889-896.

[30] Focchi, M. Strategies To Improve the Impedance Control Performance of a Quadruped Robot $P h D$ thesis, Istituto Italiano di Tecnologia, 2013.

[31] Boaventura, T., Focchi, M., Frigerio, M., Buchli, J., Semini, C., Medrano-Cerda, G. A., and Caldwell, D. G. On the role of load motion compensation in high-performance force control. IEEE/RSJ International Conference on Intelligent Robots and Systems, 2012.

[32] Colgate, E., and Hogan, N. An analysis of contact instability in terms of passive physical equivalents. IEEE International Conference on Robotics and Automation, 1989, Vol.1. pp. 404-409.

[33] Anderson, B. D. O., and Vongpanitlerd, S. Network analysis and synthesis: a modern systems theory approach Courier Dover Publications, 2006.

[34] Colgate, J. E. The control of dynamically interacting systems. PhD thesis, Massachusetts Institute of Technology, Cambridge, Massachusetts, 1986.

[35] Colgate, J. E., and Schenkel, G. G. Passivity of a class of sampleddata systems: Application to haptic interfaces. Journal of Robotic Systems, 1997, pp. 37-47.

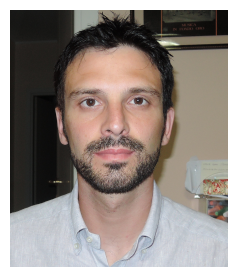

Michele Focchi is currently a Post-Doc researcher at the Advanced Robotics department of Istituto Italiano di Tecnologia. He received both the B.Sc. and the M.Sc. in Control System Engineering from Politecnico di Milano in 2004 and 2007, respectively. Until 2009 he worked for the R\&D department of Indesit company. In 2009 he 
joined the Advanced Robotic Department (ADVR) at Istituto Italiano di Tecnologia, (Genova, Italy) developing a prototype of a novel concept of air-pressure driven micro-turbine for power generation for which he obtained an international patent and several awards. In 2013 he got a Ph.D. degree in robotics, getting involved in the Hydraulically Actuated Quadruped Robot project where he developing low-level controllers for locomotion purposes. Currently his research interests range from planning of dynamic motions to whole body control.

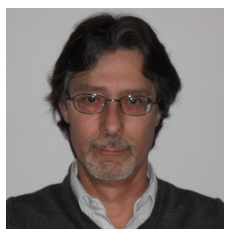

G.A. Medrano-Cerda received the B.Sc. degree in electro-mechanical engineering from the Universidad Nacional Autonoma de Mexico in 1977, and the M.Sc. and Ph.D. degrees in control systems from Imperial College, London, in 1979 and 1982, respectively. From 1982 to 1985 he was an associate professor at the Division de Estudios de Postgrado, Facultad de Ingenieria, Universidad Nacional Autonoma de Mexico. From 1985 to 1986 he was a research fellow at the Department of Engineering, University of Warwick. From 1986 to 2002 he was a lecturer at the Department of Electronic and Electrical Engineering at the University of Salford. During this period he set up the Advanced control and robot locomotion laboratory at the University of Salford. In 1999 he became a control systems consultant at Las Cumbres Observatory (formerly Telescope Technologies Ltd) and later in 2002 he joined the company as a senior control engineer pioneering work in H-infinity control system design and implementation for astronomical telescopes. Since 2009 he has been a senior research scientist at the Advanced Robotics Department, Istituto Italiano di Tecnologia. His research interests are in the areas of robust control, adaptive control, modelling and identification, fuzzy systems and advanced robotic applications, in particular to walking robots.

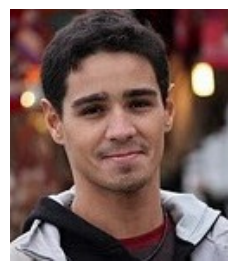

Thiago Boaventura received his B.Sc. and M.Sc. degrees in mechatronic engineering from Federal University of Santa Catarina, Florianopolis, Brazil, in 2009 and the Ph.D. degree in robotics, cognition, and interaction technologies from a partnership between Istituto Italiano di Tecnologia and University of Genoa, Genova, Italy, in 2013. He is a Postdoctoral Researcher with Agile and Dexterous Robotics Laboratory, ETH Zurich, Zurich, Switzerland. He is mainly involved in the EU FP7 BALANCE project with a focus in the collaborative impedance control of exoskeletons. His research interests include impedance and admittance control, model-based control, legged robotics, optimal and learning control, and wearable robotics.

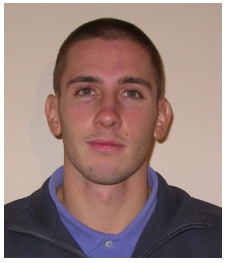

Marco Frigerio received his B.Sc. and M.Sc. degrees in computer science from the University of Milano Bicocca, respectively in 2006 and 2008, and the Ph.D. degree in Robotics from the Istituto Italiano di Tecnologia (IIT) in 2013. He is currently a Post-Doc researcher at the Dynamic Legged Systems lab of IIT, where he is involved in the development of hydraulic legged robots. His research interests include software for robotics, software architectures, kinematics and dynamics of mechanisms.

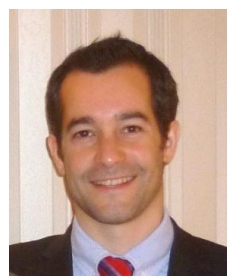

Claudio Semini is the head of the Dynamic Legged Systems lab of the Department of Advanced Robotics at Istituto Italiano di Tecnologia (IIT). He holds an MSc degree from ETH Zurich in electrical engineering and information technology (2005). From 2004 to 2006 he first visited the Hirose Lab at the Tokyo Institute of Technology, followed by work on mobile service robotics at the Toshiba R\&D center in Kawasaki, Japan. During his doctorate from 2007-2010 at the IIT he designed and constructed the quadruped robot HyQ and worked on its control. After a post-doc in the same department, in 2012, he became the head of the Dynamic Legged Systems lab. His research focus lies on the construction and control of highly dynamic and versatile legged robots in real-world environments.

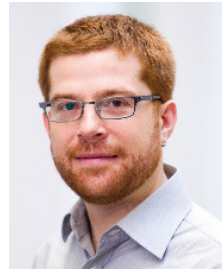

Jonas Buchli received the Diploma degree in electrical engineering from ETH Zurich, Zurich, Switzerland, in 2003 and the Ph.D. degree from Ecole Polytechnique Federale de Lausanne, Lausanne, Switzerland, in 2007. From 2007 to 2010, he was a Postdoctoral Researcher with the Computational Learning and Motor Control Laboratory, University of Southern California (USC), where he was the Team Leader of the USC Team for the DARPA Learning Locomotion challenge. From 2010 to 2012, he was a Team Leader with the Advanced Robotics Department, Istituto Italiano di Tecnologia, Genova, Italy. He is currently an Assistant Professor with the Institute of Robotics and Intelligent Systems, ETH Zurich, Zurich, Switzerland, and the Director of the Agile and Dexterous Robotics Lab. Dr. Buchli has received a Prospective and an Advanced Researcher Fellowship from the Swiss National Science Foundation (SNF). In 2012, he received a Professorship Award from the SNF.

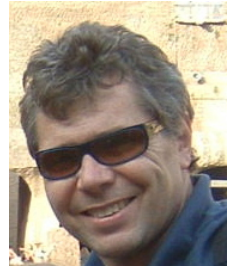

Darwin G Caldwell received the B.Sc. and $\mathrm{Ph} . \mathrm{D}$. degrees in robotics from University of Hull, Hull, U.K., in 1986 and 1990, respectively, and the M.Sc. degree in management from University of Salford, Salford, U.K., in 1996. He is the Director of Robotics with Istituto Italiano di Tecnologia, Genova, Italy. He is a Visiting/Honorary/Emeritus Professor with University of Sheffield, the University of Manchester, and University of Wales, Bangor. His research interests include innovative actuators and sensors, haptic feedback, force augmentation exoskeletons, dexterous manipulators, humanoid robotics, biomimetic systems, rehabilitation robotics, and telepresence and teleoperation procedures. 